\title{
ORIGINAL
}

\section{PLAGUICIDAS Y PCBS EN SUERO EN POBLACIÓN GENERAL DE BARAKALDO POSIBLEMENTE EXPUESTA AL HEXACLOROCICLOHEXANO ENTRE 1947 Y 2002}

\author{
Juan J Aurrekoetxea Agirre (1,2), M Begoña Zubero (1,3), Cristina Jiménez García (4), \\ Fernando Goñi Irigoyen (4,5), Koldo Cambra Contín (6), Eva Alonso Fustel (7) y M Con- \\ cepción Cadiñanos Díaz-Tejeiro (7).
}

(1) Departamento de Medicina Preventiva y Salud Pública. Universidad del País Vasco-Euskal Herriko Unibertsitatea. Leioa, Bizkaia.

(2) Subdirección de Aseguramiento y Contratación, Departamento Sanidad, Gobierno Vasco. Donostia-San Sebastián. Gipuzkoa.

(3) Unidad de Investigación. Hospital de Cruces, Barakaldo, Bizkaia.

(4) Laboratorio de Salud Pública. Donostia-San Sebastián. Gipuzkoa.

(5) Ciber Epidemiología y Salud Pública (CIBERESP)

(6) Centro de Investigación Biomédica. Pamplona. Navarra.

(7) Subdirección de Salud Pública, Departamento Sanidad, Gobierno Vasco. Bilbao. Bizkaia.

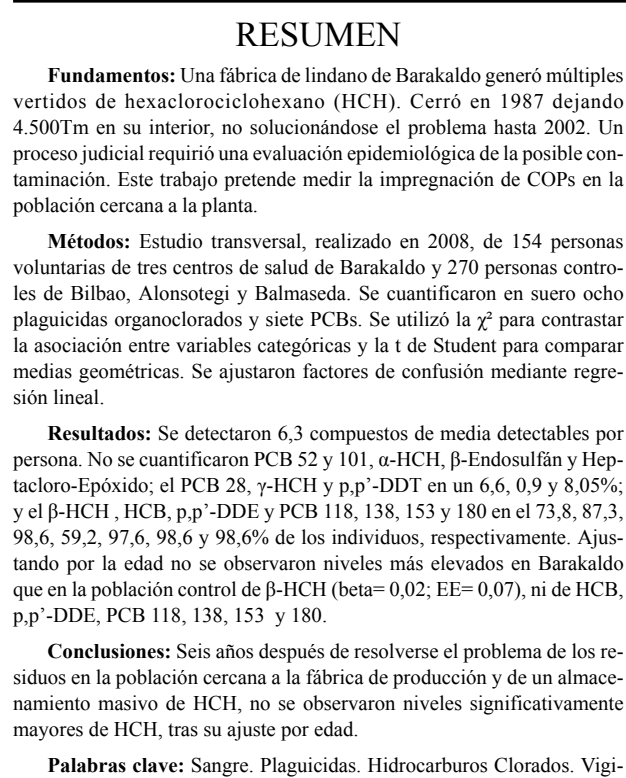
ancia de la población. Población Urbana. Exposición Ambiental.

Juan José Aurrekoetxea.

Departamento de Medicina Preventiva y Salud Pública

Universidad del País Vasco-Euskal Herriko Unibertsitatea.

Barrio Sarriena s/n. Leioa, Bizkaia.

Correo electrónico: jj.aurreko@gmail.com

\section{ABSTRACT \\ Exposure to Lindane, other Pesticides and Organochlorines in the General Population Barakaldo, Spain}

Bakcground: A plant of lindane in Barakaldo produced discharges of
hexachlorocyclohexane (HCH). Closed in 1987 leaving inside $4500 \mathrm{Tm}$;
the problem was not solved until 2002. A judicial process required an epi-
demiological assessment of the possible contamination The aim of this
study is to measure the degree of impregnation of the POPs in the nearby
population.

Methods: Cross-sectional study conducted in 2008, 154 volunteers from three health centres in Barakaldo and 270 controls from Bilbao, Alonsotegi and Balmaseda. We measured serum levels of eight organochlorine pesticides, and seven polychlorinated biphenyls (PCBs). We used the $\chi^{2}$ test to study the association between categorical variables and $\mathrm{t}$ test of Student to compare geometric means. Confounding factors were adjusted using a linear regression model.

Results: There was a detectable compound average of 6.3 per person. PCBs 52 and 101, $\alpha$-HCH, $\beta$-endosulfan and heptachlor epoxide could not be quantified; PCB 28, $\gamma$-HCH and p, p'-DDT were detected in 6.6, 0.9 and $8.05 \%$ of subjects respectively and the $\beta-\mathrm{HCH}, \mathrm{HCB}, \mathrm{p}, \mathrm{p}$-DDE, and PCBs $118,138,153$ and 180 in the 73.8, 87.3, 98.6, 59.2, 97.6, 98.6 y 98.6 $\%$ respectively. After adjusting for age, there were no higher levels of $\beta$ $\mathrm{HCH}$ in Barakaldo than in controls, (beta $=0.02, \mathrm{SE}=0.07$ ), or HCB, p, p'DDE, PCB 118, PCB 138, PCB 153 and PCB 180.

Conclusion: Six years after having solved the problem near to the factory and storage of $\mathrm{HCH}$, there was no significantly higher levels of $\mathrm{HCH}$, after adjustment for age.

Key words: Blood. Pesticides. Chlorinated hydrocarbons. Population surveillance. Urban population. Environmental exposure. 


\section{INTRODUCCIÓN}

Los plaguicidas son sustancias químicas destinadas a proteger principalmente los cultivos, luchando contra diferentes organismos, contra insectos, roedores, etc. Pueden tener utilidad en salud pública en la lucha contra los vectores de enfermedades. El hecho de tratarse de biocidas les supone a priori una potencial toxicidad. Los plaguicidas organoclorados, junto con los bifenil policlorados (PCBs) y otras sustancias halogenadas presentan una alta persistencia en el medio ambiente. La utilización de biomarcadores para valorar la exposición de la población a los contaminantes ambientales es una alternativa cada vez más utilizada ${ }^{1}$. Desde que en 2004 entró en vigor en España el Convenio de Estocolmo sobre compuestos orgánicos persistentes (COPs) han sido diversas las experiencias llevadas a cabo en nuestro país para cuantificar, mediante biomarcadores, el grado de exposición de la población general no expuesta a fuentes concretas de estos contaminantes ${ }^{2}$. La fuente de exposición más importante a estos compuestos es la laboral, en la manufactura y aplicación de los plaguicidas, y para los PCBs el contacto con fluidos dieléctricos en condensadores y transformadores, aunque afectan a una muy pequeña parte de la población. En población general la fuente principal de aporte de estos compuestos son los alimentos ${ }^{3}$.

La alta persistencia en la grasa de los plaguicidas organoclorados y otros COPs hace que se acumulen en la cadena alimentaria, en el contenido graso de los alimentos. Los plaguicidas organoclorados más frecuentemente detectados en humanos son el diclorodifeniltricloroetano ( $\mathrm{p}, \mathrm{p}^{\prime}$-DDT) y su metabolito el diclorodifenildicloroetileno (p,p'-DDE), el hexaclorobenceno (HCB) y el beta-hexaclorociclohexano $(B-H C H)^{4}$. Que el $\beta-\mathrm{HCH}$ se detecte más frecuentemente que sus isómeros $\alpha-\mathrm{HCH}$ ó $\gamma-\mathrm{HCH}$ sería debido a que éstos se metabolizan y eliminan antes en nuestro organismo ${ }^{5}$, así como en las plantas y animales que nos sirven de alimento, persistiendo el isómero $ß$ $\mathrm{HCH}$ en nuestra alimentación. El Departamento de Sanidad del Gobierno Vasco realizó mediciones de residuos de plaguicidas en los alimentos en el período 1990-1995. Las ingestas fueron muy bajas y representaron en todos los casos porcentajes de las Ingestas Diarias Tolerables inferiores al 7\%. Los grupos que más plaguicidas organoclorados aportaban a la dieta fueron los alimentos ricos en grasa de origen animal: pescados, huevos, leche y productos lácteos ${ }^{6}$.

En España el uso plaguicidas organoclorados se restringió fuertemente en la década de los 70. Los Convenios de Rotterdam y Estocolmo, de 1998 y 2001 respectivamente, llevaron a minimizar la exposición a los COPs. A pesar de ello, hoy en día persisten en el ambiente y en los tejidos humanos ${ }^{7}$. Un estudio realizado sobre muestras representativas de la población general de las islas Canarias, mostró niveles elevados de DDT y DDE en suero, 171 y 262 ng/g lípido, respectivamente, en mayores de 20 años $^{8}$ y para $\gamma-\mathrm{HCH}$ con niveles de 73 y $69 \mathrm{ng} / \mathrm{g}$ lípido para hombres y mujeres, respectivamente $^{9}$. Otro estudio realizado sobre muestras representativas de la población de Cataluña ${ }^{10}$ mostró valores más moderados: 140 para HCB, $83 \beta-\mathrm{HCH}, 4 \gamma-\mathrm{HCH}, 23$ DDT y $424 \mathrm{DDE}$ ng/g lípido, así como una media de 247 ng/g lípido para la suma de los PCBs más frecuentes, PCB 118, 138, 153 y 180. Este estudio podría servir de referencia del nivel de COPs en suero que podemos esperar en población española no expuesta a fuentes adicionales concretas de COPs. En la provincia de Bizkaia la única experiencia existente es un estudio realizado en población general adulta voluntaria de cuatro zonas. Mostró medias de 79 para HCB, 43 para $\beta-\mathrm{HCH}, 19$ para DDT y 191 para DDE $\mathrm{ng} / \mathrm{g}$ lípido y $244 \mathrm{ng} / \mathrm{g}$ lípido para la suma de PCBs. El $\gamma$-HCH únicamente fue detectable en un $3,5 \%$ de la muestra ${ }^{11}$. Un estudio de Andalucía mostró medias 18, 14 y 501 ng/g lípido para $\gamma-\mathrm{HCH}$, DDT y DDE respectiva- 
mente 7 . Otro estudio de interés realizado en España fue el realizado por el EPIC, que mostró COPs de muestras de suero recogidas entre 1992 y 1995 con niveles netamente más elevados de HCB, $\beta$-HCH, DDT y DDE, 379, 167, 44 y 822 ng/g lípido, respectivamente $^{12}$, y de la suma de los PCBs más frecuentes, con $459 \mathrm{ng} / \mathrm{g}$ lípido ${ }^{13}$.

Entre 1947 y 1987 se fabricó lindano técnico en una empresa de la Vega de Ansio, en el barrio de Lutxana de Barakaldo. Por cada tonelada fabricada del isómero $\gamma-\mathrm{HCH}$ o lindano, el único con valor comercial como insecticida, se generaban ocho no comerciales, compuestas por el resto de los isómeros. La empresa fue acumulando o vertiendo estos residuos de $\mathrm{HCH}$ no comercial en diferentes emplazamientos. En 1987, tras la prohibición por el Servicio Vasco de Salud a la empresa de trasladar los residuos fuera de sus instalaciones comenzó a almacenarlos en su interior, donde acumularon 4.500 toneladas de $\mathrm{HCH}$ no comercial. Tras el cierre de la empresa por parte de su dirección, la Viceconsejería de Medio Ambiente del Gobierno Vasco se hizo cargo de la gestión de los residuos almacenados, así como de los vertidos existentes. Entre 1999 y 2001 una empresa creada para tal efecto fabricó triclorobenceno y oxicloruro de cobre a partir de los residuos de $\mathrm{HCH}$ puro en el mismo solar donde se había fabricado el HCH. Las tierras contaminadas de 26 emplazamientos de vertidos con 412.000 metros cúbicos fueron almacenadas en una empresa siderúrgica de Barakaldo, ya cerrada y cercana a la anterior y confinadas en 2002 en una celda de seguridad.

Pese a haber transcurrido más de 20 años desde el cierre de la empresa y 6 desde la finalización de las actividades de eliminación de residuos y limpieza de la zona, se suscitó preocupación en la población de Barakaldo por la posible exposición al $\mathrm{HCH}$, por su persistencia y por su toxicidad. Este estudio analizó los niveles de ocho plaguicidas organoclorados, $\mathrm{HCB}, \alpha-\mathrm{HCH}, \beta-$
HCH, $\gamma$-HCH, p,p'-DDE y p,p'-DDT, heptacloro epóxido y $\beta$-endosulfán, así como de los PCBs más frecuentes, PCB 28, 52, 101, $118,138,153$ y 180.

\section{SUJETOS Y MÉTODO}

Población y muestra. El diseño se correspondió con un estudio transversal, realizado entre las persoans residentes en distintas poblaciones próximas a la Vega de Ansio de Barakaldo y lejanas a la misma. La población próxima a la planta consistió en residentes del entorno supuestamente expuesto, donde se situó la planta de fabricación primero y transformación de $\mathrm{HCH}$ después, en la Vega de Ansio, población con la asistencia sanitaria cubierta por el Servicio Vasco de Salud-Osakidetza en los Centros de Salud de Zuazo, La Paz y Lutxana. Se estableció que la muestra estuviera compuesta por personas sin patología crónica renal o hepática que acudían a extracción de sangre para analítica, con un mínimo de residencia en Barakaldo de 7 años, es decir, viviendo desde el año 2000 y con edades comprendidas entre 20 y 70 años. El objetivo fue captar un mínimo de 40 personas por centro de salud, un mínimo total de 120 individuos. Un estudio previo de Bizkaia mostraba en población no expuesta en su trabajo a plaguicidas $^{11}$ una media geométrica de $\beta$ $\mathrm{HCH}$ de 41,9 ng/g lípido. Era necesario un tamaño de las muestras de estudio y de referencia de 103 individuos para que una diferencia de $20 \mathrm{ng} / \mathrm{g}$ lípido fuera significativa, cifra muy inferior a la observada en España. para población general de $167 \mathrm{ng} / \mathrm{g}$ lípido ${ }^{12}$. Aceptaron la participación en el estudio y donaron una muestra de sangre un total de 154 personas, 45 de Lutxana, 48 de La Paz y 61 de Zuazo. Además, se solicitó la participación de las personas que trabajaron en la planta de transformación de $\mathrm{HCH}$. Los seis individuos que lo hicieron de manera fija en la planta accedieron a participar en el estudio. La fase de campo con las tomas de muestras y encuestas se realizó a finales de 2008. 
La población de referencia fue una muestra de individuos de municipios de Bizkaia alejados de la Vega de Ansio. Se trataba de un total de 270 personas con edades entre 20 y 70 años de edad y residentes de los municipios de Balmaseda y Alonsotegi, dos núcleos semiurbanos de baja y alta presencia industrial, respectivamente, y los barrios de Santutxu y Rekalde de Bilbao, dos zonas urbanas no industriales, y que formaban parte de otro estudio, disponiéndose en ese momento de muestras de sangre recogidas ese mismo año en las mismas condiciones. Habían sido seleccionados para evaluar la posible exposición a los contaminantes de una incineradora situada en Bilbao, metales pesados ${ }^{14}$, dioxinas, furanos y $\mathrm{PCBs}^{15}$ y plaguicidas organoclorados ${ }^{11}$. Se seleccionó a los individuos de manera aleatoria a partir del censo municipal cedido por los ayuntamientos, completándose con voluntarios del barrio o municipio y, por último, voluntarios que acudían al centro de salud para extracción de sangre. La diferente procedencia de la muestra pudo condicionar las diferencias en su composición en cuanto a otras variables, como la edad, nivel de estudios, ocupación, clase social o consumo de productos de huerta (tabla 1). El Comité de Ética de Investigación Clínica de Euskadi dio el correspondiente informe favorable para la realización de este estudio.

Toma de muestras. A todos los participantes en el estudio se les extrajo $10 \mathrm{ml}$ de sangre. Las muestras fueron tomadas, tratadas, almacenadas y transportadas en las condiciones establecidas por los laboratorios de referencia ${ }^{16}$. Se midieron los niveles de ocho plaguicidas organoclorados: $\mathrm{HCB}, \alpha-\mathrm{HCH}$, $\beta$-HCH, $\gamma$-HCH, p,p'-DDE, p,p'-DDT, heptacloro epóxido y $\beta$-endosulfán. Asimismo, por razones de oportunidad, se cuantificaron los niveles de los PCBs 28, 52, 101, 118, 138 153 y 180 , COPs todos ellos de origen industrial.

Determinaciones analíticas. El análisis se realizó en el Laboratorio de Salud Pública de Gipuzkoa, siguiendo un método previamente descrito ${ }^{17}$. El límite de cuantificación (LQ) de los PCBs fue de $0,10 \mathrm{ng} / \mathrm{ml}$, mientras que el del $\beta$-HCH y del p,p'-DDE fue de $0,20 \mathrm{ng} / \mathrm{ml}$ y el del resto de los plaguicidas de $0,30 \mathrm{ng} / \mathrm{ml}$. A los individuos con valores de biomarcadores inferiores al límite de cuantificación, para el análisis estadístico se les asignó la mitad del mismo, antes de su corrección por lípidos. Los resultados finales se refirieron al contenido en lípido, determinado enzimáticamente, y se expresaron en nanogramo por gramo de lípido (ng/g lípido). Las determinaciones analíticas de las dos zonas de estudio se realizaron simultáneamente y de manera ciega. El laboratorio carecía de información de la procedencia de las muestras.

Variables de estudio. Todos los participantes cumplimentaron un cuestionario que recogió información sobre su edad, sexo, zona geográfica de su domicilio, índice de masa corporal (IMC), variables socioeconómicas: nivel de estudios, situación laboral, y consumos de tabaco y de alimentos de granja o huerta locales.

Análisis estadístico. Para la descripción de niveles de plaguicidas se obtuvieron las medias geométricas e intervalos de confianza al 95\% de probabilidad (IC 95\%), dado que la transformación logarítmica de las variables las aproximaba a la distribución normal y reducía su variabilidad. Las variables con más del $80 \%$ de los individuos con niveles por debajo del límite de cuantificación se analizaron únicamente de manera cualitativa, utilizando la prueba de la $\chi^{2}$ como contraste de hipótesis. Las diferencias de medias se contrastaron mediante la prueba de la t de Student. El análisis de comparación de medias entre los valores observados en Barakaldo y en la zona de referencia se realizó agrupando a todos los individuos de la localidad. La comparación de medias para una variable cualitativa ordinal se contrastó mediante la prueba de la varianza para gradiente lineal. Para evaluar qué variables se 
asociaban de manera significativa e independiente con los PCBs y plaguicidas organoclorados se utilizó un modelo de regresión lineal múltiple. En el modelo se incluyó siempre la variable zona de muestreo, perteneciente a Barakaldo o no, y se introdujeron por el método parsimonioso el resto de las variables recogidas en el cuestionario. Se contrastó el gradiente lineal de las variables ordinales. Se estimó la varianza explicada por el modelo mediante el coeficiente de determinación, $\mathrm{R}^{2}$. El análisis estadístico se realizó mediante el paquete estadístico SPSS versión 16.0 .

\section{RESULTADOS}

La tabla 1 muestra las características de los 154 individuos procedentes de la zona próxima al recinto industrial que compusieron la muestra de Barakaldo y los 270 individuos de la zona de referencia. Se observó una media de edad de 53,5 años en la muestra de Barakaldo, frente a la de 45,8 en la población de referencia $(\mathrm{p}<0,001)$. El IMC no mostró diferencia de medias $(\mathrm{p}=0,255)$, pero sí una mayor proporción de individuos con sobrepeso en Barakaldo $(p=0,036)$. Se observó que en Barakaldo los individuos presentaban con mayor frecuencia estudios medios, bachiller o formación profesional $(p<0,001)$. En la zona de referencia había mayor proporción de trabajadores activos que en la de Barakaldo, 68 vs 51\% ( $\mathrm{p}=0,004)$ y un consumo de productos de huerta más frecuente, 31 vs $11 \%$, respectivamente $(\mathrm{p}<0,001)$. No se observaron diferencias estadísticas en cuanto a sexo $(p=0,198)$ o consumo de tabaco $(p=0,327)$.

La tabla 2 muestra los individuos con niveles detectables de COPs, PCBs o plaguicidas. No se observó en ningún individuo niveles cuantificables de los PCBs 52 y 101 y de los plaguicidas $\alpha-\mathrm{HCH}$, heptacloro epóxido y $\beta$-endosulfán. El PCB 28 se detectó en 28 individuos $(6,6 \%)$, el $\gamma$ - $\mathrm{HCH}$ en 4 $(0,9 \%)$ y el p,p'-DDT en $34(8,0 \%)$. Todos estos contaminantes quedaron excluidos del análisis estadístico ulterior. Los PCBs 118 , 138,153 y 180 fueron cuantificables en 251 individuos $(59,2 \%), 414(97,6 \%), 418$ $(98,6 \%)$ y $418(98,6 \%)$, respectivamente. Asimismo, los plaguicidas HCB, ß-HCH y p,p'-DDE se detectaron en 370 individuos $(87,3 \%), 313(73,8 \%)$ y $418(98,6 \%)$, respectivamente. Únicamente se observó un significativo mayor número de muestras cuantificables de $\mathrm{B}-\mathrm{HCH}$ en Barakaldo respecto a la población de referencia. La tabla 3 muestra cuántos COPs fueron cuantificables en el suero a los individuos de la muestra. No se observó diferencia significativa entre la media de COPs observada en el grupo de Barakaldo, media 6,3 COPs (IC 95\%: 6,1$6,5)$ y la de la población control 6,3 COPs (IC 95\%: 6,1-6,5) $(\mathrm{p}=0,998)$.

La tabla 4 compara las medias observadas de los COPs con más del $20 \%$ de individuos con valores cuantificables, mostrando valores medios de $\mathrm{B}-\mathrm{HCH}$ significativamente más elevados en Barakaldo 47,4 ng/g lípido, que en la zona de referencia, $35,5 \mathrm{ng} / \mathrm{g}$ lípido $(\mathrm{p}=0,001)$. Asimismo, la media de $\mathrm{p}, \mathrm{p}^{\prime}-\mathrm{DDE}$ en Barakaldo, 211,6 ng/g lípido, era más elevada que la de la población control, 178,8 ng/g lípido, con valores próximos al límite de significación $(\mathrm{p}=0,065)$. El HCB, así como los PCBs cuantificables más frecuentes, PCBs 118, 138, 153 y 180, mostraron valores más elevados en Barakaldo, no significativos estadísticamente

La tabla 5 muestra la relación de los cuatro PCBs más frecuentes y de su sumatorio con las variables que se asociaron de manera significativa con ellos en el modelo de regresión lineal y en el que se mantuvo la zona de vivienda, Barakaldo o control. No se observó interacción significativa alguna y únicamente la edad, el sexo o el IMC, además de la variable de estudio, la zona de vivienda, mostraron alguna asociación estadística significativa. Los individuos de Barakaldo mostraron, una vez ajustados sus valores por el resto de variables, niveles significativamente más bajos de PCBs que los del grupo 


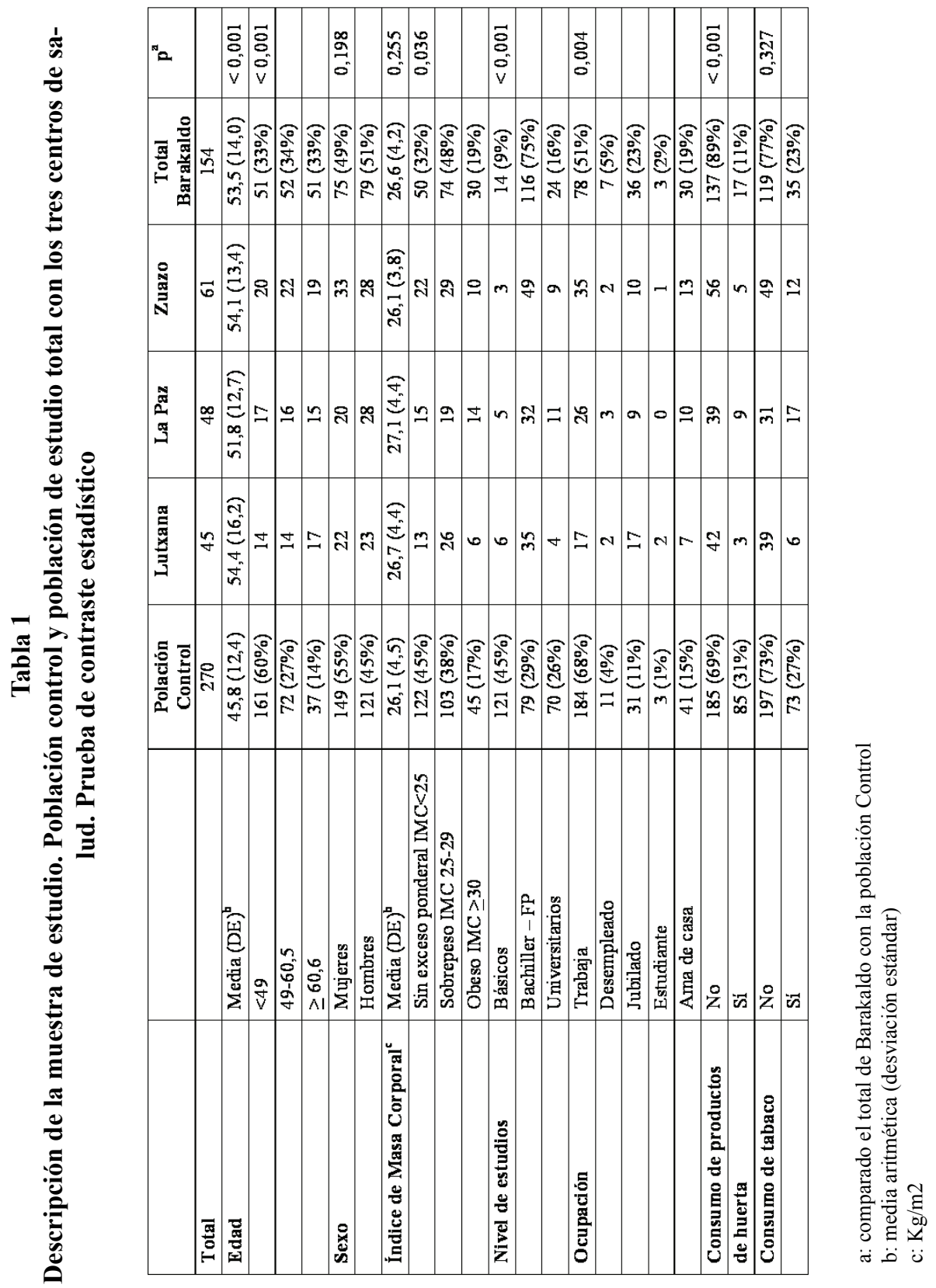




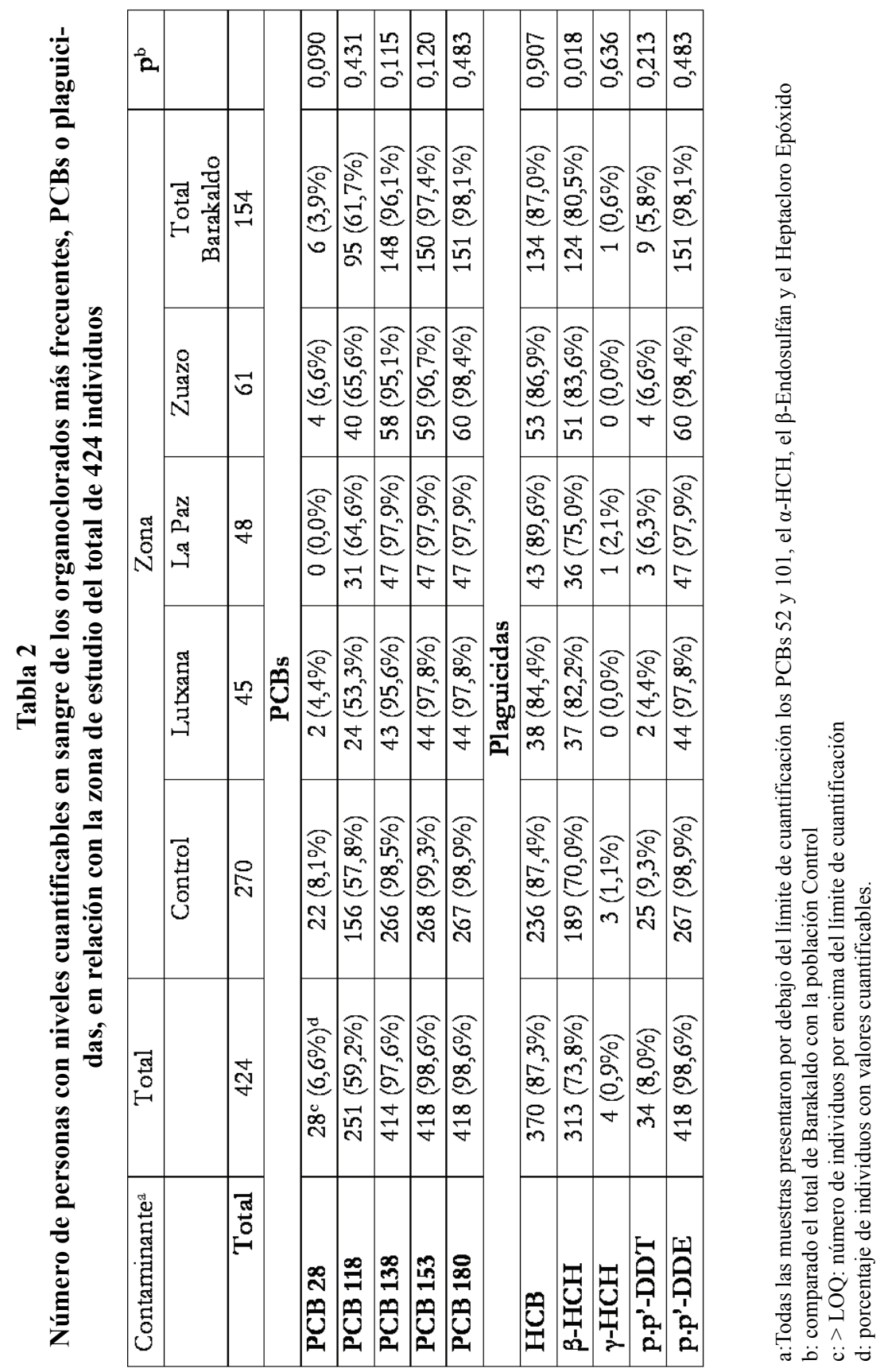


Tabla 3

Número de COPs cuantificables en cada individuo del total de Barakaldo y de la zona de referencia. Diferencia de medias no significativa $(p=0,998)$

\begin{tabular}{|c|c|c|c|c|c|c|c|c|c|}
\hline $\mathrm{N}^{0} \mathrm{de}$ & \multicolumn{3}{|c|}{ Total } & \multicolumn{3}{|c|}{ Barakaldo } & \multicolumn{3}{|c|}{ Control } \\
\hline & $\mathbf{N}$ & $\%$ & $\%^{a}$ & $\mathbf{N}$ & $\%$ & $\%^{a}$ & $\mathbf{N}$ & $\%$ & $\%^{a}$ \\
\hline 1 & 3 & 0,7 & 0,7 & 1 & 0,6 & 0,6 & 2 & 0,7 & 0,7 \\
\hline 2 & 3 & 0,7 & 1,4 & 3 & 1,9 & 2,6 & 0 & 0,0 & 0,7 \\
\hline 3 & 5 & 1,2 & 2,6 & 2 & 1,3 & 3,9 & 3 & 1,1 & 1,9 \\
\hline 4 & 40 & 9,4 & 12,0 & 13 & 8,4 & 12,3 & 27 & 10,0 & 11,9 \\
\hline 5 & 51 & 12,0 & 24,1 & 11 & 7,1 & 19,5 & 40 & 14,8 & 26,7 \\
\hline 6 & 87 & 20,5 & 44,6 & 33 & 21,4 & 40,9 & 54 & 20,0 & 46,7 \\
\hline 7 & 179 & 42,2 & 86,8 & 78 & 50,6 & 91,6 & 101 & 37,4 & 84,1 \\
\hline 8 & 50 & 11,8 & 98,6 & 12 & 7,8 & 99,4 & 38 & 14,1 & 98,1 \\
\hline 9 & 6 & 1,4 & 100 & 1 & 0,6 & 100 & 5 & 1,9 & 100 \\
\hline Total & 424 & 100 & - & 154 & 100 & - & 270 & 100 & - \\
\hline
\end{tabular}

a: Porcentaje acumulado

de referencia $(\mathrm{p}<0,001$; para todos ellos). La edad mostró una asociación constante con los cuatro contaminantes, con mayores niveles de PCBs a mayor edad ( $\mathrm{p}_{\text {gradiente }}<$ $0,001)$. Los hombres presentaron niveles de PCB 138, 153 y 180 más elevados que las mujeres ( $\mathrm{p} \leq 0,001$; para todos ellos). Los obesos presentaron niveles significativamente más elevados de PCB 118 ( $\mathrm{p}=$ $0,003)$, mientras que los valores de PCB 180 disminuyeron con el aumento de la masa corporal $\left(\mathrm{p}_{\text {gradiente }}<0,001\right)$. El resto de variables, consumo de tabaco o productos de huerta local, ocupación, clase social o educación no se asociaron de manera significativa con los PCBs ( $p>0,05)$. Los modelos explicaban entre un 40,5 y el 50,5 $\%$ de la variabilidad de los PCBs.

Las variables asociadas a los plaguicidas organoclorados, HCB, $\beta-\mathrm{HCH}$ y p,p'DDE, así como con el número de COPs observados en el suero de cada individuo, fueron las mismas que para los PCBs (tabla 6), aunque no siempre en el mismo sentido. Tampoco mostraron asociación estadística el consumo de tabaco o de pro- ductos de huerta local, la ocupación, la clase social o el nivel educativo. Su poder predictivo fue más bajo para el p,p'-DDE $(26,1 \%)$ encontrándose comprendido entre el 40,6 y el 49,9\% para el resto de plaguicidas, valores próximos a los observados para los PCBs. Los individuos de Barakaldo presentaron niveles significativamente más bajos de $\mathrm{HCB}(\mathrm{p}=0,002)$ y un menor número de COPs detectables en su suero $(p<0,001)$, no mostrando diferencias para el $\beta-H C H(p=0,799)$ y el $p, p '-$ DDE $(p=0,589)$. La edad también mostró asociación con las cuatro variables, con mayores niveles de contaminación y mayor número de contaminantes a mayor edad ( $\left.\mathrm{p}_{\text {gradiente }}<0,001\right)$. Las mujeres mostraron niveles más elevados de HCB y $\beta$ $\mathrm{HCH}(\mathrm{p}<0,001$; para ambos). El IMC, por último, mostró un gradiente significativo con los tres plaguicidas y con el número de COPs detectables; a más sobrepeso mayores niveles y número de contaminantes detectables $\left(\mathrm{p}_{\text {gradiente }}<0,001\right)$. El $ß-\mathrm{HCH}$ muestra un comportamiento diferente en las tablas 4 y 6 . La tabla 7 muestra las medias geométricas del $\beta-\mathrm{HCH}$ en la 


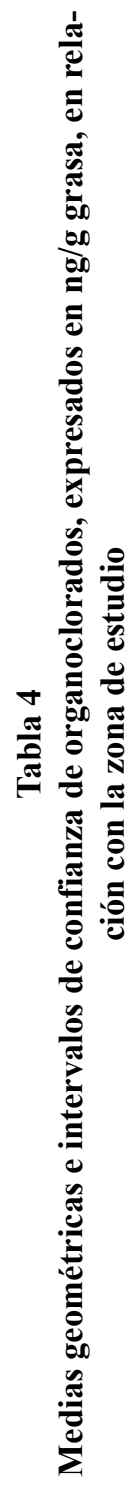

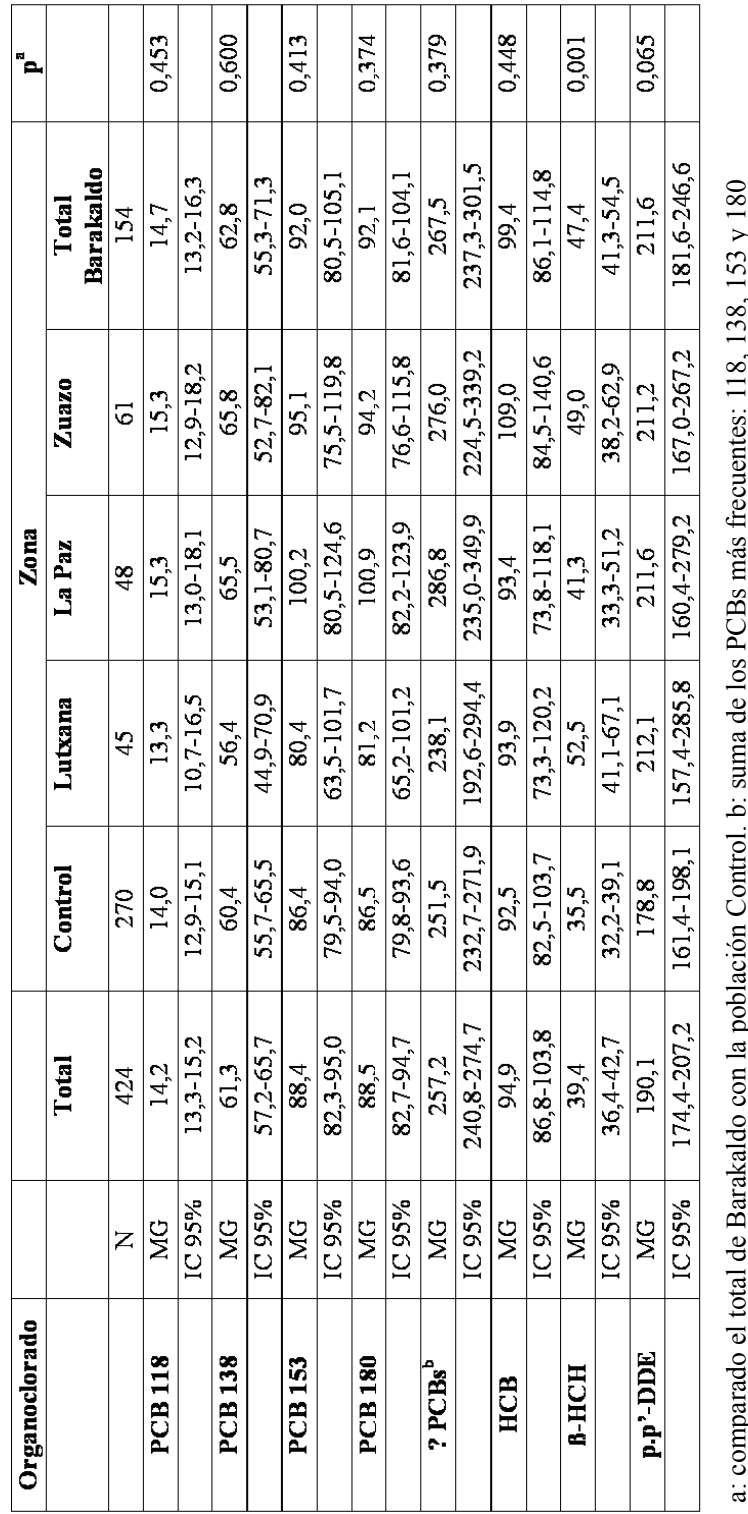




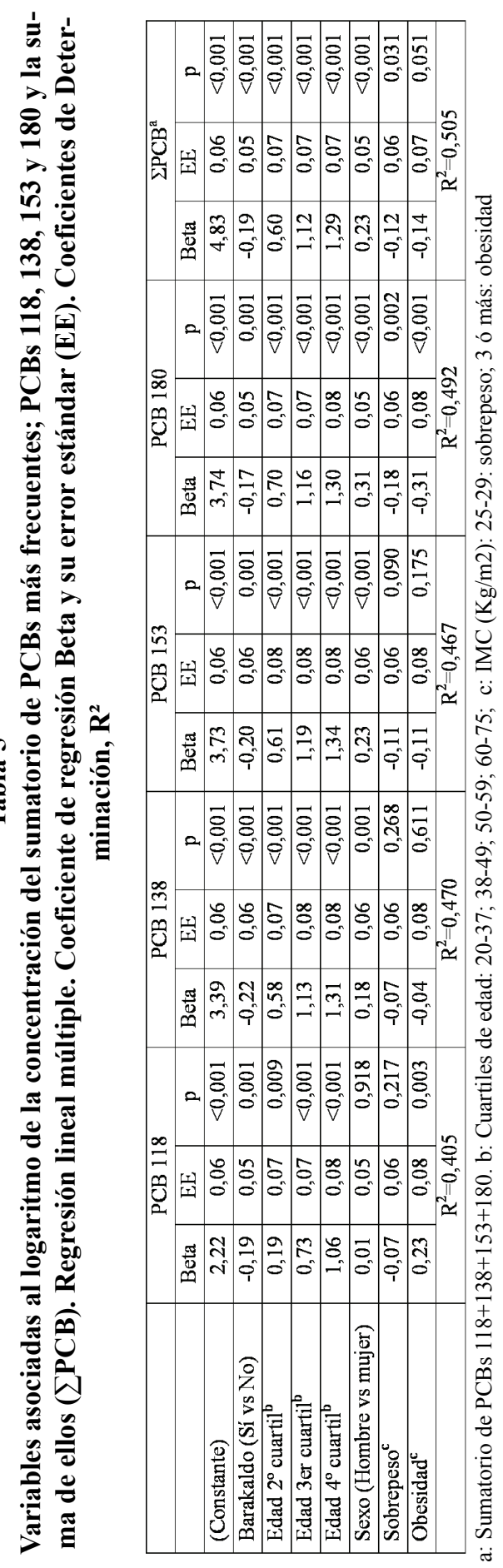

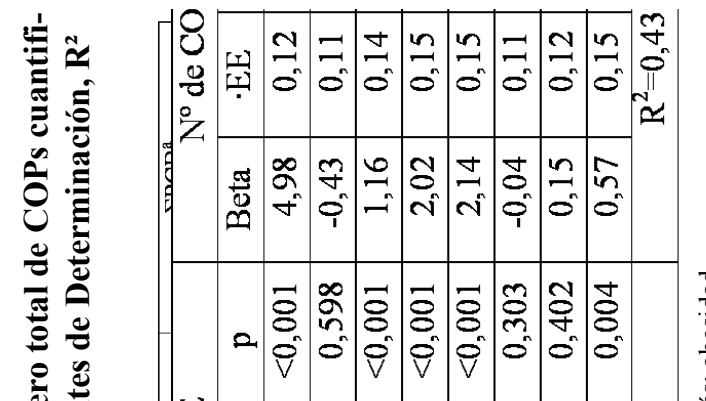

葡

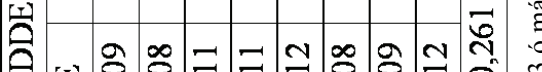

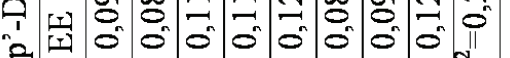

ส

苞

可

อิ

ㅎํㄹํํㄹ

궁

풍

6

尩

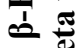

คิ

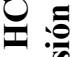

告

尝 2 $\widetilde{\sim}$

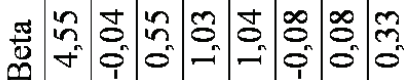

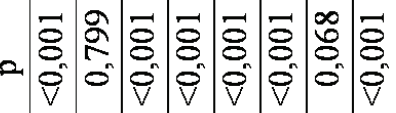

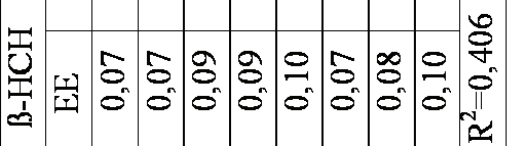

๘

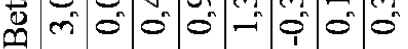

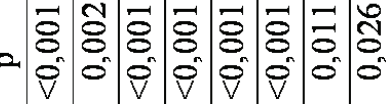

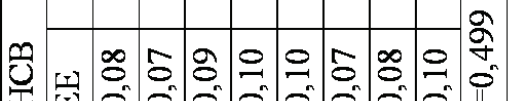

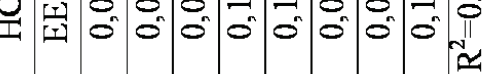

ฐิกี

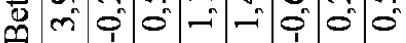

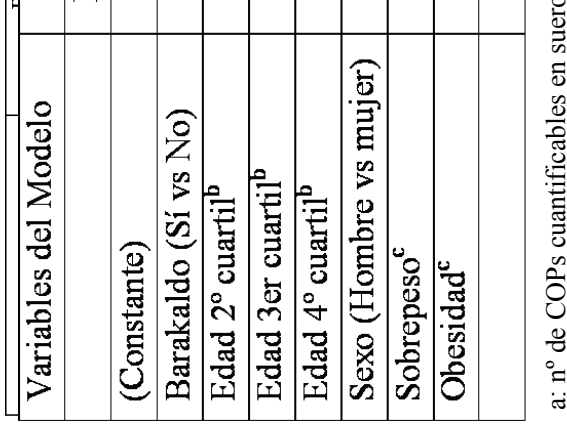

Rev Esp Salud Pública 2011, Vol. 85, N. ${ }^{\circ} 2$ 
Tabla 7

Medias geométricas de ß-HCH y sus intervalos de confianza en Barakaldo y la población de referencia estratificadas según los tertiles de edad de la muestra global

\begin{tabular}{|l|c|c|c|c|c|c|c|}
\hline & \multicolumn{3}{|c|}{ Referente } & \multicolumn{3}{c|}{ Barakaldo } & $\mathrm{P}$ \\
\hline Tertiles de edad & $\mathrm{N}$ & $\mathrm{MG}$ & $\mathrm{IC} 95 \%$ & $\mathrm{~N}$ & $\mathrm{MG}$ & $\mathrm{IC} 95 \%$ & \\
\hline$<40$ años & 107 & 20,0 & $18,3-21,9$ & 35 & 22,2 & $18,2-27,2$ & 0,294 \\
\hline $40-56,4$ años & 97 & 41,0 & $35,5-47,4$ & 43 & 46,0 & $37,6-56,2$ & 0,383 \\
\hline$? 56,5$ años & 66 & 72,5 & $61,2-86,0$ & 76 & 68,4 & $56,3-83,2$ & 0,665 \\
\hline \multicolumn{1}{|c|}{$\mathrm{p}^{\mathrm{a}}$} & & $<0,001$ & & & $<0,001$ & & \\
\hline
\end{tabular}

a: $\mathrm{p}$ de tendencia lineal

población de Barakaldo y la de referencia, estratificando por tertiles de edad. Las medias de $\mathrm{B}-\mathrm{HCH}$ no mostraron diferencias significativas en relación con la zona geográfica tras su estratificación, aunque sí un claro gradiente con la edad en ambas zonas $(\mathrm{p}<0,001)$. La distribución de individuos de las dos zonas fue significativamente diferente, con una mayoría de casos de Barakaldo en el tertil más alto de edad y de la población de referencia en el primer tertil, $\chi^{2}=28,43(\mathrm{p}<0,001)$.

Entre los seis trabajadores de la planta de transformación de HCB no hubo valores detectables de PCBs 52 y 101 ni de $\alpha$ $\mathrm{HCH}, \gamma-\mathrm{HCH}$, heptacloro epóxido y $\beta$ endosulfán. En uno se pudo cuantificar PCB 28 y en dos de ellos el PCB 118 y el p,p'-DDT. El HCB se detectó en cinco trabajadores y los PCBs 138-153-180 y los plaguicidas $\mathrm{HCB}, \beta-\mathrm{HCH}$ y $\mathrm{p}, \mathrm{p}$ '-DDE se cuantificaron en los seis trabajadores. Se observó una media de PCB 118 de 15,5 ng/g lípido (IC 95\%: 8,6-27,8); PCB 138 de 91,6 ng/g lípido (58,8-142,8); PCB 153 de $146,7 \mathrm{ng} / \mathrm{g}$ lípido $(95,0-226,6)$; PCB 180 de 135,0 ng/g lípido 91,2-199,7); HCB de 67,4 ng/g lípido (29,7-152,8); ß$\mathrm{HCH} 249,5 \mathrm{ng} / \mathrm{g}$ lípido $(112,4-553,8)$; p,p'-DDE 364,6 ng/g lípido (140,2$947,9) ; y$ p,p'-DDT de 31,3 ng/g lípido $(21,9-44,9)$. No se observaron diferencias significativas al comparar estas medias con las del resto de individuos del estudio $(\mathrm{p}>0,05)$ salvo para el $\mathrm{B}-\mathrm{HCH}$, que mostró niveles significativamente más altos entre los que habían trabajado en la planta $(\mathrm{p}<0,001)$.

\section{DISCUSIÓN}

El estudio muestra, tras ajustar por edad y otros factores de confusión, que la población de Barakaldo no mostraba diferencias significativas en relación con el número y los niveles de COPs en suero respecto a la población de referencia, alejada de las instalaciones de la planta de fabricación y posteriormente de transformación del $\mathrm{HCH}$. Los resultados de la población de Barakaldo se encuentran dentro del rango de los encontrados en otras poblaciones no expuestas a fuentes concretas de COPs. La significación estadística fue menor para todas las sustancias cuando se comparó la población control con los barrios de Barakaldo, por lo que no se mostraron estos valores. Los trabajadores de la planta de transformación de $\mathrm{HCH}$ mostraron una media de $\beta-\mathrm{HCH}$ significativamente más elevada que la del global de la muestra estudiada, aunque sin incremento para $\alpha-\mathrm{HCH}$ o $\gamma-\mathrm{HCH}$. La diferencia significativa en el número de muestras con valores detectables de $\beta-\mathrm{HCH}$ y en las medias observadas entre la población control y la de Barakaldo era explicable por factores de confusión, principalmente la edad. Se trata de un contaminante altamente persistente y la muestra de Barakaldo era 7,8 años mayor 
que la de la población control. Esta diferencia de edad, así como la del resto de variables sociodemográficas, se podría explicar por la diferente procedencia de las personas de la muestra, debido a que en la población control principalmente se captó a partir del censo, lo cual pudo condicionar su menor edad, menor grado de obesidad y mayor nivel de estudios. Tras el ajuste por la edad y otras covariables en el modelo de regresión múltiple no se observaban diferencias significativas en los niveles de $\beta-\mathrm{HCH}$. La estratificación por edad mostraba la diferente distribución de los dos grupos, consecuencia del sesgo introducido al utilizar métodos diferentes de muestreo en las dos zonas de estudio. El muestreo de base poblacional a partir del censo supone un enorme esfuerzo y lleva a una escasa participación de los individuos, alejándose del objetivo de la representatividad. El muestreo en el Centro de Salud durante la toma de muestras de análisis requiere de la colaboración de los trabajadores, las enfermeras principalmente, pero la colaboración de los individuos es muy elevada. Queda la limitación de la dudosa representatividad de esa muestra de la población general. En este estudio medimos compuestos tóxicos a muy bajas concentraciones, lejos de poder estar asociados con la enfermedad.

En población general, exceptuando a los individuos laboralmente expuestos, se ha considerado a la dieta como la fuente principal de aporte de estas sustancias ${ }^{18}$. Los tres plaguicidas con presencia mayoritaria en los sueros de los individuos de este estudio fueron el HCB, el $\beta-H C H$ y el p,p'-DDE, que junto al p,p'-DDT son los más frecuentemente detectados ${ }^{4}$. Los resultados obtenidos para población general en este estudio son comparables a los observados en Bizkaia ${ }^{11} \mathrm{y}$ en Cataluña ${ }^{10}$. En el sur de España ${ }^{7}$ y en las islas Canarias ${ }^{8,9}$ se observaron niveles de plaguicidas más elevados. Muestras obtenidas con anterioridad en cinco provincias españolas también mostraron niveles de plaguicidas $^{12}$ y $\mathrm{PCBs}^{13}$ más elevados. Entre estas cinco provincias se encontraba Gipuzkoa, que no tendría fuentes de exposición a COPs diferentes a las de Bizkaia, lo que sugeriría una tendencia decreciente de la concentración de contaminantes en el organismo.

Dentro del panorama internacional, tienen especial interés los resultados de los muestreos de los CDC para los Estados Unidos, con muestras del orden de 2.000 individuos, tanto en 1999 como en 2001. Sus valores medios eran inferiores a los de este estudio y en muchos casos por debajo del límite de detección para los PCBs y los plaguicidas, salvo para el p,p'-DDE, que mostraba 297 y $338 \mathrm{ng} / \mathrm{g}$ lípido, respectivamente. Ello sugiere una menor exposición a PCBs y plaguicidas que en nuestro medio, salvo una exposición al p,p'-DDT algo alejada en el tiempo, dada la eliminación del organismo de este contaminante y quedando su metabolito más persistente, el p,p'-DDE. Se considera que la presencia de p,p'-DDE tiene su origen en la dieta $y$, en menor medida, en el metabolismo del p,p'-DDT en el organismo del ser humano ${ }^{19}$.

No se observan en este estudio diferencias significativas en los niveles de compuestos organoclorados según zona de estudio, salvo para el sumatorio de PCBs y HCB, que mostraron niveles significativamente inferiores en Barakaldo que en la población control tras el ajuste de los factores de confusión. Los valores de PCBs y de los tres plaguicidas analizados, p,p'-DDE, HCB y $\beta-\mathrm{HCH}$, aumentaron con la edad, incluso tras el ajuste por otras variables. El hecho de que se trate de sustancias lipofílicas y bioacumulables explica esta asociación ${ }^{20}$. Los niveles medios de $\mathrm{HCB}$ y el $\beta-\mathrm{HCH}$ fueron superiores en mujeres que en hombres, al igual que lo observado por Jakszyn ${ }^{13}$ y Porta $^{10}$ y en contra de lo observado en Portugal ${ }^{21,22}$. Esta disparidad sugiere que no se trate de diferencias en el metabolismo, sino a diferencias en la dieta. La mayor concentración de PCBs en hombres de este estudio, por el contrario, es 
habitualmente referida ${ }^{10,13}$, por lo que podría tratarse de exposición laboral antigua, además de a la dieta.

La obesidad se asoció positivamente con los niveles de HCB, ß-HCH y p,p'-DDE, a mayor masa corporal mayores niveles, así como para el PCB 118, pero de manera inversa con el PCB 180. La asociación inversa entre los PCBs y la masa corporal ha sido ya descrita por otros estudios ${ }^{23-25}$. Wolf ${ }^{23}$ sugiere que la mayor cantidad de grasa en los obesos supondría un factor de dilución en la fase de exposición al COP y, sin embargo, que en exposiciones alejadas en el tiempo la metabolización y eliminación de los organoclorados sería más lenta. En todo caso, el IMC debería tenerse siempre en cuenta al valorar la exposición a sustancias lipofílicas ${ }^{26}$. Los cambios bruscos de peso y la lactancia podrían modificar la carga corporal y la concentración de los COPs.

Los niveles de plaguicidas no mostraron asociación con la ocupación, la clase social o el consumo de productos de huerta o granja locales o el de tabaco. Ello apunta a que la fuente de exposición más probable sea alimentaria, de procedencia muy diversa y con escasa contribución local, ya que los mayores consumidores de productos de granjas locales solamente consumían productos de huerta de temporada y huevos pero con una frecuencia muy baja. Se acepta que para la mayor parte de la población nos encontramos ante una exposición difusa y transmitida por los alimentos, principalmente ${ }^{18}$. El estudio de dieta y cáncer, EPIC, observó una asociación significativa entre la concentración de PCBs y el consumo de pescado ${ }^{13}$. La población vasca se caracteriza por su elevado consumo de pescado, lo cual podría explicar la mayor presencia de PCBs observada en este estudio en relación con los de otras procedencias. Un estudio de dieta total mostró para el periodo 1999-2000 que la ingesta media del Total de Sustancia con Actividad Dioxina (TSAD) de la población de la Comunidad Autónoma del País Vasco fue de 2.6 WHO-TEQ pg/kg/día, un $60 \%$ menor respecto a lo observado cinco años antes. El alimento que más contribuía era el pescado, siendo los no-orto PCBs, los compuestos que más contribuían al TSAD ${ }^{27}$. También se estudiaron los residuos de plaguicidas en los alimentos. Las ingestas a través de alimentos fueron muy bajas y representaron en todos los casos porcentajes de las correspondientes Ingestas Diarias Tolerables inferiores al 7\% y se mantuvieron bajas durante todo el periodo 1990-1995. Los grupos que más plaguicidas organoclorados aportaron fueron los de origen animal: pescados, huevos, leche y productos lácteos ${ }^{6}$. El EPIC, sin embargo, no observó asociación entre los niveles de plaguicidas y el consumo de alimentos en la actualidad ${ }^{12}$. Un estudio sueco observó que los niveles de HCB, $\beta$ HCH y p,p'-DDE en la edad maternal se asociaron con el consumo de pescado en su adolescencia. Ello nos lleva a pensar que los niveles actuales de plaguicidas organoclorados u otros COPs son reflejo de exposiciones antiguas $^{25}$. Este estudio muestra que las variables introducidas en los modelos de regresión explicaban menos del $50 \%$ de la variabilidad de los COPs analizados. Ello es explicable porque no se introducen en los mismos factores asociados realmente con la exposición, sino variables relacionadas con la persistencia de los mismos en el organismo, como sexo, edad, IMC, etc. En poblaciones expuestas a fuentes de exposición concretas, laborales o ambientales, mejoraría la varianza explicada. La dieta en población general explica una pequeña parte de la mis$\mathrm{ma}^{12-13}$. Ello lleva a pensar que la población general se encuentra expuesta a estas sustancias a niveles difusos de baja intensidad ${ }^{10}$.

Entre los trabajadores de la planta de transformación de $\mathrm{HCH}$ se observó una media de $\beta-\mathrm{HCH}$ significativamente más elevada que el del global de la muestra estudiada. Por el contrario, no presentaron diferencias significativas en relación con otros plaguicidas o PCBs. La observación de niveles más altos de $\beta-\mathrm{HCH}$ es consecuente con que sea éste el isómero del $\mathrm{HCH}$ más persistente 
y que aún no haya sido del todo eliminado. $\mathrm{Su}$ valor medio, sin ajustar por lípidos se encontraría por encima de los obtenidos para población general, no expuesta a fuentes concretas de $\mathrm{B}-\mathrm{HCH}$, en otros estudios, salvo los de Portugal ${ }^{21,22}$. Cabe mencionar, no obstante, que los trabajadores de esta planta extremaron las medidas higiénicas para minimizar la exposición al $\mathrm{HCH}$. En todo caso, no se detectaban los isómeros $\alpha-\mathrm{HCH}$ ó $\gamma-\mathrm{HCH}$, isómeros menos persistentes que el $\beta-\mathrm{HCH}^{4}$. A diferencia de lo que ocurre con el DDE que es metabolito del DDT, y en contra de lo que se pensaba ${ }^{28}$, el lindano no se isomeriza o transforma en $\alpha-\mathrm{HCH}$ o $\beta-\mathrm{HCH}^{29}$.

Los resultados de este estudio indican que la población general adulta de Barakaldo, villa muy industrializada hasta hace poco tiempo y con la presencia de una empresa de fabricación de $\mathrm{HCH}$, se encuentra expuesta actualmente a PCBs y plaguicidas organoclorados en los mismos niveles que otras poblaciones adultas de Bizkaia. Los plaguicidas y PCBs más persistentes en el organismo no muestran mayores niveles en esta población que en las poblaciones de referencia. El hecho de que el isómero del $\mathrm{HCH}$ más persistente, el $\beta-\mathrm{HCH}$, no muestre mayor nivel en Barakaldo que en la población control indica que no ha habido mayor grado de exposición a este contaminante ni recientemente ni a medio plazo derivado de focos puntuales o locales y que la fuente principal de exposición es común, apuntando a la alimentación. Entre los trabajadores de la planta de transformación del $\mathrm{HCH}$ se observó que los niveles de $\mathrm{B}-\mathrm{HCH}$ eran significativamente superiores al resto de la población estudiada. La observación de un valor significativamente elevado de $\beta$ $\mathrm{HCH}$ apunta a que estas personas aún no han eliminado la totalidad de este plaguicida. Para el resto de contaminantes analizados no se observan diferencias estadísticamente significativas entre los trabajadores y la población general.

\section{AGRADECIMIENTOS}

Los autores de este estudio agradecen a las Direcciones de las Comarcas de Atención Primaria del Servicio Vasco de Salud, Osakidetza, Comarca Ezkerraldea-Enkarterri y Comarca Bilbao, a los responsables de los Centros de Salud y a sus trabajadores, por la colaboración prestada $\mathrm{y}$, especialmente, a los participantes en el estudio que de manera voluntaria colaboraron amablemente en el estudio.

\section{BIBLIOGRAFÍA}

1.Angerer J, Ewers U, Wilhelm M. Human biomonitoring: State of the art. Int J Hyg Environ Health. 2007;210:201-228.

2. Porta M, Puigdomènech E, Ballester F. Nuestra contaminación interna: Concentraciones de compuestos tóxicos persistentes en la población española. Madrid: Catarata;2009.

3 .Porta M, Kogevinas M, Zumeta E, Sunyer J, RibasFito N. Concentraciones de compuestos tóxicos persistentes en la población española: el rompecabezas sin piezas y la protección de la salud pública. Gac Sanit. 2002; 16:257-66.

4.World Health Organization. Health risks of persistent organic pollutants from lon-range transboundary air pollution. The Regional Office for Europe of the World Health Organization, Copenhagen. Denmark, 2003.

5. Hayes WJ. Chlorinated hydrocarbons insecticides. En: Hayes WJ, Lawes ER, editors. Pesticides studied in Man. San Diego:Academic Press, 1991:731-868.

6.Jalón, M Urieta, I Macho ML, Azpiri M. Vigilancia de la Contaminación Química de los Alimentos en la Comunidad Autónoma del País Vasco: 1990-1995. Servicio central de publicaciones. Gobierno Vasco. Vitoria-Gasteiz, 1997. [citado 24 de agosto de 2007]. Disponible en: www.osanet.euskadi.net/r85-20339/es/contenidos/informacion/sanidad_alimentaria/es_1247/seguridad.html

7.Cerrillo I, Olea-Serrano MF, Ibarluzea J, Exposito J, Torne P, Laguna J, et al. Environmental and lifestyle factors for organochlorine exposure among women living in Southern Spain. Chemosphere. 2006;62:191724.

8.Zumbado M, Goethals M, Alvarez-Leon EE, Luzardo OP, Cabrera F, Serra-Majem Ll, et al. Inadvertent expo- 
sure to organochlorine pesticides DDT and derivatives in people from the Canary Islands (Spain). Sci Total Environ. 2005;339:49-62.

9.Luzardo OP, Goethals M, Zumbado M, Alvarez-León EE, Cabrera F, Serra-Majem L, et al. Increasing serum levels of non-DDT-derivative organochlorine pesticides in the younger population of the Canary Islands (Spain). Sci Total Environ. 2006;367:129-38.

10.Porta $M$, Gasull $M$, Puigdomènech $E$, Garí $M$, de Basea MB, Guillén M, et al. Distribution of blood concentrations of persistent organic pollutants in a representative sample of the population of Catalonia. Environ Int. 2010;36:655-64.

11.Zubero MB, Aurrekoetxea JJ, Ibarluzea JM, Goñi F, López R, Etxeandia A, et al. Plaguicidas organoclorados en población general adulta de Bizkaia. Gac Sanit. 2010;24:274-81.

12.Jakszyn P, Goñi F, Etxeandia A, Vives A, Millán E, López R, et al.Serum levels of organochlorine pesticides in healthy adults from five regions of Spain. Chemosphere. 2009;76:1518-24.

13.Agudo A, Goñi F, Etxeandia A, Vives A, Millán E, López R, Amiano P, et al. Polychlorinated biphenyls in Spanish adults: Determinants of serum concentrations. Environ Res. 2009;109,620-8.

14.Zubero MB, Aurrekoetxea JJ, Ibarluzea JM, Arenaza MJ, Rodríguez C, Sáenz JR. Heavy metal levels (Pb, $\mathrm{Cd}, \mathrm{Cr}$ and $\mathrm{Hg}$ ) in the adult general population near an urban solid waste incinerator. Sci Total Environ. 2010;408:4468-74.

15.Zubero MB, Ibarluzea JM, Aurrekoetxea JJ, Rivera J, Parera J, Abad E, et al. Serum levels of chlorinated dibenzodioxins and dibenzofurans (PCDD/F) and PCBs in general population living near to an urban waste treatment plant in Biscay, Basque Country. Chemosphere. 2009;76:784-91.

16.Patterson DG, Isaacs SG, Alexander LR, Turner WE, Hampton L, Bernert JT, et al. Method 6, determination of specific polychlorinated dibenzo-p-dioxins and dibenzofurans in blood and adipose tissue by isotope dilution-high-resolution Mass Spectrometry. IARC Sci Publ. 1991;108:299-342.

17.Goñi F, Lopez R, Etxeandia A, Millan E, Amiano P. High throughput method for the determination of organochlorine pesticides and polychlorinated biphenyls in human serum. J Chromatogr B Analyt Technol Biomed Life Sci. 2007;852:15-21.

18.Hanaoka T, Takahashi Y, Kobayashi M, Sasaki S, Usuda M, Okubo S, et al. Residuals of beta-hexachlo- rocyclohexane, dichlorodiphenyltrichloroethane, and hexachlorobenzene in serum, and relations with consumption of dietary components in rural residents in Japan. Sci Total Environ. 2002;286:119-27.

19.Centers for Disease Control and Prevention. Department of Health and Human Services. National Center for Environmental Health. Division of Laboratory Sciences. Third National Report on Human Exposure to Environmental Chemicals. Atlanta, Georgia 303413724. NCEH Pub. No. 05-0570. July 2005.

20.Jaga K, Dharmani Ch. Global Surveillance of DDT and DDE levels in human tissues. Int J Occup Environ Health. 2003;16:7-20.

21.Cruz S, Lino CM, Silveira MI. Evaluation of organochlorine pesticide residues in human serum from an urban and two rural populations in Portugal. Sci Total Environ. 2003;317:23-35.

22.Lino CM, da Silveira MI. Evaluation of organochlorine pesticidas in serum from students in Coimbra, Portugal: 1997-2001. Environ Res. 2006;102:339-51.

23. Wolff MS, Britton JA, Teitelbaum SL, Eng S, Deych $\mathrm{E}$, Ireland $\mathrm{K}$, et al. Improving organochlorine biomarker models for cancer research. Cancer Epidemiol Biomarkers Prev. 2005;14,2224-36.

24.J Ibarluzea, M Alvarez-Pedrero, M Guxens, L Santa Marina, M Basterrechea, A Lertxundi, et al.. Sociodemographic, reproductive and dietary predictors of organochlorine compounds levels in pregnant women in Spain. Chemosphere. 2011;82:114-120

25.Glynn A, Aune M, Darnerud PO, Cnattingius S, Bjerselius R, Becker W, et al. Determinants of serum concentrations of organochlorine compounds in Swedish pregnant women: a cross-sectional study. Environ Health. 2007;6:2.

26.Carreño J, Rivas A, Granada A, Lopez-Espinosa MJ, Mariscal M, Olea N, et al. Exposure of young men to organochlorine pesticides in Southern Spain. Environ Res. 2007;103:55-61.

27.Cuervo L. Ingesta de dioxinas y dioxin-like PCBs a través de los alimentos en la CAPV. Resultados del estudio de la dieta total en el periodo 1999-2000. Salud Pública. Osasun Publikoa. Bol Epidemiol Comunidad Autónoma País Vasco. 2002;14:5-6. Disponible en: www.euskadi.net/r33-2288/es/contenidos/informacion/boletin_epidem/es_5482/adjuntos/14bolecast.pdf.

28.Jensen AA. Chemical contaminants in human milk. Residue Rev. 1983;89:1-128. 
29.CECOP. Comité de Examen de los Contaminantes Orgánicos Persistentes. Naciones Unidas. Programa de las Naciones Unidas para el Medio Ambiente. Segunda reunión. Examen de los proyectos de perfil del riesgo sobre: Lindano. UNEP/POPS/POPRC.2/10. Ginebra, 2006. [citado 7 de agosto de 2006]. Disponible en: www.pops.int/documents/meetings/poprc/POPRC2/P OPRC2_doc_s/POPRC2_doc_10_s.doc. 